\title{
Experimental Characterization of Damped CFRP Materials with an Application to a Lightweight Car Door
}

\author{
Alessandro Fasana, Alessandro Ferraris, Andrea Giancarlo Airale, \\ Davide Berti Polato, and Massimiliana Carello
}

Politecnico di Torino, Turin, Italy

Correspondence should be addressed to Alessandro Ferraris; alessandro.ferraris@polito.it

Received 12 May 2017; Accepted 19 July 2017; Published 24 August 2017

Academic Editor: Feng Zhu

Copyright (c) 2017 Alessandro Fasana et al. This is an open access article distributed under the Creative Commons Attribution License, which permits unrestricted use, distribution, and reproduction in any medium, provided the original work is properly cited.

\begin{abstract}
This paper presents a complete design procedure for defining a dynamic model of a Carbon Fibre Reinforced Polymer (CFRP) component with an embedded damping material layer. The experiment to determine the mechanical characteristics of the materials is performed by the Oberst beam technique to provide precise material properties for a Finite Element (FE) model. The technique implemented, namely, the Linear Identification by Polynomial Expansion in the Z-domain (LIPEZ) method, is used to compare the experimental data with the numerical simulation results provided by the modal parameters to be compared with the numerical results. Two automotive components (a leaf spring and an outer shell of front door) have been tested. The research revealed the utter importance of a correct definition of the geometry for the numerical models. Finally, the positive effects for acoustic performance with a thin layer of KRAIBON ${ }^{\circledR}$ SUT9609/24 damping material, included in the stacking sequence of the CFRP component, are highlighted.
\end{abstract}

\section{Introduction}

Recently, car buyers have raised their expectation on vehicle comfort with respect to the past, when performances and reliability were sufficient to set the quality of the vehicles. Vehicle electrification in this contest emphasizes the Noise and Vibration Harshness (NVH) because of the absence of the thermal engine noise and the more wide use of lightweight material [1-4]. Carbon fibre reinforced plastic (CFRP) materials provide good mechanical characteristics and promote light structures but emphasized $\mathrm{NVH}$ at the same time. For this reason, the use of damping materials in passive constrained layer configuration is often taken into consideration [5] to lower the vibrational response of CFRP structures, but such procedure increases both the weight and the manufacturing process. A precise numerical model, for example, a finite element model (FEM) of a structure, is only reliable if material properties are correctly defined, so that Section 2 deals with the use of the so-called Oberst test method [6-8] to define Young modulus, loss factor of damping, and structural materials. These properties have been calculated through the analysis of the frequency response of tested samples, measured at different temperatures in controlled climatic condition. As expected, the CFRP properties do not change while the damping material and the result show a quite typical viscoelastic behaviour.

With the aim of defining a complete design procedure for CFRP (automotive) components with an embedded damping layer, the comparison of the numerical (from a FEM) and the measured mode shapes (from experimental modal analysis) is the most feasible and diffused procedure $[9,10]$. Over the last few decades, a number of papers dealing with the problem of modal parameters estimation of vibrating structures have been presented; [11-13] are good examples. The linear identification by polynomial expansion in the Zdomain (LIPEZ) method adopted in this paper starts from the rational fraction polynomials (RFP) representation of the frequency response function (FRF) and expounds a total least square method in the Z-domain [14]. The procedure is briefly presented in Section 3. In particular, the method is applied [15] to two automotive components which are also reproduced at simulation level with computational FEM 


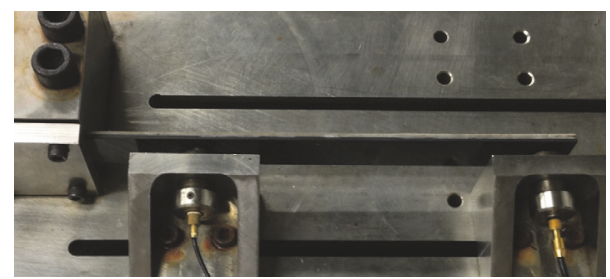

FIGURE 1: The Oberst beam test bench.

simulation in order to perform a correlation analysis. The chosen components include a leaf spring (Section 4) with simple geometry and a door panel (Section 5) for its influence in vehicle global NVH characteristics. Both components are made with structural CFRP, whose mechanical properties have been examined in Section 2 using the Oberst beam test method.

\section{CFRP and Damped Sandwich Characterization}

This section describes the procedure adopted to measure Young's modulus and the loss factor of two different materials: a set of pure T300 epoxy, twill CFRP [0/90/0] specimens, and a set of 5 layers sandwich specimens with the following staking sequence [0/90] CFRP + 1 Layer KRAIBON SUT9609/24 + [0/90] CFRP structure combining interlaminar damping material with 2 layers of CFRP T300. The activity aims at defining the characteristics of the materials, which is going to be introduced in the material card of a FE simulation.

The Oberst beam test is a standard method [6] to characterize laminated materials and basically consist in a clampedfree beam as shown in Figure 1. The beam is excited by a contactless electromagnetic transducer, which exerts a sweptsine force. The input force frequency range depends on the type of specimen under test. Another contactless capacitive transducer is located at the tip of the beam to detect the output response (velocity): the spectrum of the output identifies the natural frequencies of the beam and its loss factor. In its simplest form the identification procedure is based on the $-3 \mathrm{~dB}$ method (or half-power method), but more objective results can be achieved by implementing a least square fitting of the spectrum. By analyzing the natural frequencies with Bernoulli-Euler beam model Young's modulus can be determined.

Tests have been performed in a thermally controlled environment at different temperatures, from $-20^{\circ} \mathrm{C}$ to $+60^{\circ} \mathrm{C}$ with steps of $10^{\circ} \mathrm{C}$. The first natural frequency has always been discarded, since it can be too much affected by the imperfect constraint conditions [2]. Three samples of each material have been aged for $(250,500$, and $750 \mathrm{~h})$ and then tested to establish the effect of aging on damping capabilities of the interlaminar material. Table 1 gives the mean characteristics of the three samples, while Figure 2 presents the average variation of Young's modulus and loss factor with frequency. The CRFP T300 (Figure 2) has very stable properties and very low damping $(<1 \%)$ while the sandwich configuration-CFRP T300 + KRAIBON SUT9609/24 (Figure 3)-shows the typical

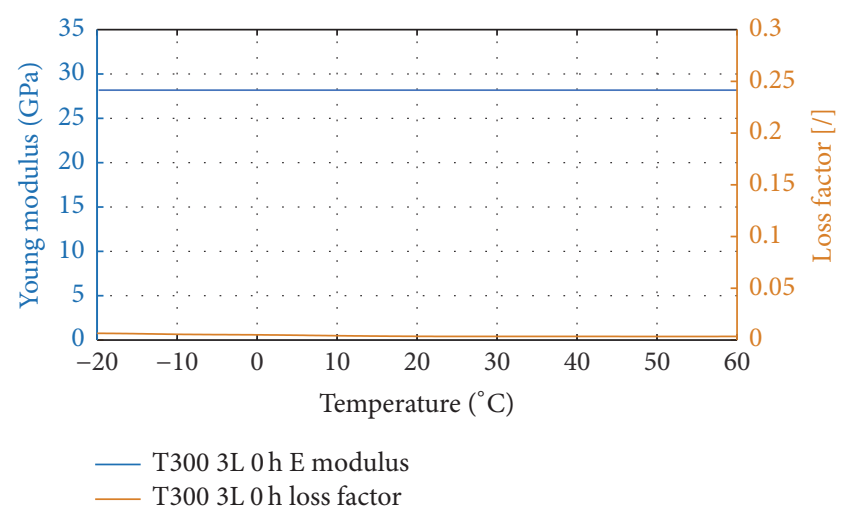

FIGURE 2: Material characteristics as a function of temperature: CFRP T300 material.

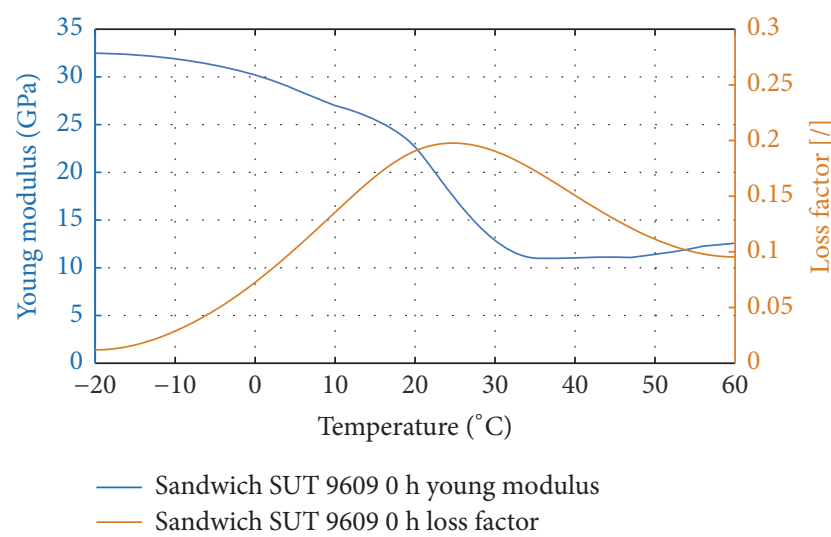

FIGURE 3: Material characteristics as a function of temperature: T300 + KRAIBON SUT9609/24 sandwich.

variations of viscoelastic materials and very good damping in the whole temperature range $0-30^{\circ} \mathrm{C}$.

The definition of the mechanical properties of these materials paves the way to their application to two test cases: a CFRP leaf spring and a CFRP car door panel, with or without a damping material in the constrained layer configuration. The numerical results obtained by a FEM have been compared to experimental results in terms of natural frequencies and mode shapes, extracted by the LIPEZ method.

\section{Outline of the LIPEZ Method}

The LIPEZ method is a frequency domain modal parameter extraction technique, which takes advantage of the Ztransform formulation. The procedure is briefly summarized in this section but its complete description is found in [14].

For a linear and time invariant system with $\mathrm{n}$ degrees of freedom, the FRF can be expressed by

$$
H_{k}=\sum_{r=1}^{2 n} A_{r} \frac{z_{k}}{z_{k}-z_{r}}
$$


TABLE 1: Mean characteristics of the samples.

\begin{tabular}{lccccc}
\hline Sample & Layers & Thickness $(\mathrm{mm})$ & Length $(\mathrm{mm})$ & Mass $(\mathrm{kg})$ & Density $\left(\mathrm{kg} / \mathrm{m}^{3}\right)$ \\
\hline CFRP T300 & 3 & 0.91 & 259.88 & 0.00393 & 1305 \\
KRAIBON SUT9609/24 & $3+1+3$ & 1.72 & 259.75 & 0.0074 & 1308 \\
\hline
\end{tabular}

where $H_{k}=H\left(\Omega_{k}\right)$ is the generic spectral line of the FRF, evaluated at frequency:

$$
\Omega_{k}=(k-1) \Delta \Omega=(k-1) 2 \pi \Delta f=\frac{\pi f_{s}(k-1)}{N-1},
$$

where $f_{s}$ is the sampling frequency, $\Delta f$ is the frequency resolution, $N$ is the number of spectral lines, and $k=1, \ldots$, $N$.

The terms related to the $Z$-transform are

$$
\begin{aligned}
& z_{r}=e^{s_{r} \Delta t}, \\
& z_{k}=e^{i(k-1) \Delta \Omega \Delta t}=e^{i \pi(k-1)(N-1)},
\end{aligned}
$$

where $i=\sqrt{-1}$ and the poles $s_{r}$ are linked to the natural angular frequencies $\omega_{r}$ and damping ratios $\xi_{r}$ by the expression $s_{r}=-\xi_{r} \omega_{r}+i \omega_{r} \sqrt{1-\xi_{r}^{2}}$.

The sum in (1) can be converted in the following rational fraction expression:

$$
H_{k}=\frac{b_{1} z_{k}+\cdots+b_{2 n} z_{k}^{2 n}}{a_{0}+a_{1} z_{k}+\cdots+a_{2 n-1} z_{k}^{2 n-1}+z_{k}^{2 n}},
$$

where the $4 n$ unknown coefficients $a_{0}, \ldots, a_{2 n-1}$ and $b_{1}, \ldots$, $b_{2 n}$ are real valued. Equation (4) can be written for $N$ spectral lines and for many FRFs (namely, NFRF) to get an overdetermined linear system of equations:

$$
\left[\begin{array}{c}
\mathbf{A}_{\mathbf{1}} \\
\vdots \\
\mathbf{A}_{\mathrm{NFRF}}
\end{array}\right] \mathbf{a}+\left[\begin{array}{ccc}
-\mathbf{B} & \cdots & \mathbf{0} \\
\vdots & \ddots & \vdots \\
\mathbf{0} & \cdots & -\mathbf{B}
\end{array}\right]\left\{\begin{array}{c}
\mathbf{b}_{\mathbf{1}} \\
\vdots \\
\mathbf{b}_{\mathrm{NFRF}}
\end{array}\right\}=\left\{\begin{array}{c}
\mathbf{w}_{\mathbf{1}} \\
\vdots \\
\mathbf{w}_{\mathrm{NFRF}}
\end{array}\right\},
$$

where $\mathbf{a}=\left[\begin{array}{llll}a_{0} & a_{1} & \cdots & a_{2 n-1}\end{array}\right]^{\mathrm{T}}$ and $\mathbf{b}_{\mathbf{m}}=$ $\left[\begin{array}{llll}b_{1} & b_{2} & \cdots & b_{2 n}\end{array}\right]_{\mathrm{m}}^{\mathrm{T}}(m=1, \ldots, \mathrm{NFRF})$ are the vectors to be determined. The matrix in (5) can surely be solved but the procedure can be time-consuming, especially when large data sets are analyzed (NFRF $\gg 1$ ) and $\mathrm{n}$ has to vary (e.g., to define a stabilization chart). A much quicker least square procedure can indeed be implemented to limit, at first, the solution to vector a:

$$
\mathbf{R a}=\mathbf{r} .
$$

The real, square $(2 n \times 2 n)$, and well-conditioned matrix $\mathbf{R}$ contains the information of all the measured FRFs but a system of only $2 n$ equations has to be solved. The poles $s_{r}=$ $\ln z_{r} / \Delta t$ can then be obtained by using the equation:

$$
a_{0}+a_{1} z+\cdots+a_{2 n-1} z^{2 n-1}+z^{2 n}=0 .
$$

Any vector $\mathbf{b}_{m}$, the related modal constants $A_{r}$, and eventually the mode shapes can then be recovered from (4).

An open source version of the implemented method can be obtained from the authors under the CC BY license.

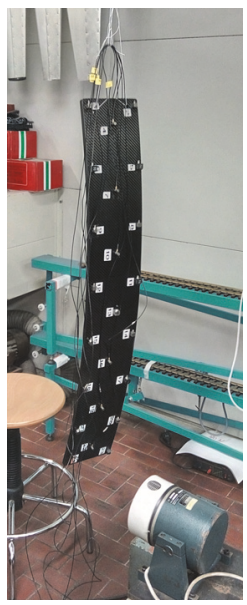

FIgURE 4: The leaf spring component: the experimental configuration with 23 accelerometers.

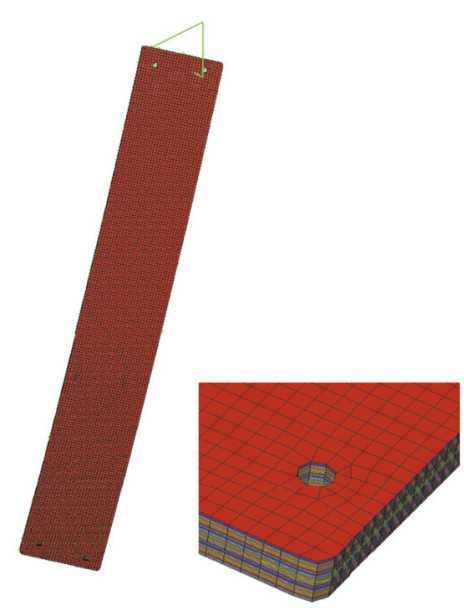

FIGURE 5: The leaf spring component: the finite element model, with a detailed view.

\section{Leaf Spring Application}

This section is devoted to the comparison of the numerical simulation and experimental results of a CRFP leaf spring formed by 31 layers of Epoxy CFRP T300, twill $2 \times$ $2240 \mathrm{gr} / \mathrm{m}^{2}$, following the stacking sequence $\left[0 /(0 / 45)_{14} / 0 /\right.$ $0]$.

4.1. Experimental Tests. The test aims at computing the modal parameters of the system in the free-free condition, with the component vertically suspended by elastic supports as shown in Figure 4 or by a Single Point Constrain (green in Figure 5). The leaf spring was excited by an electromagnetic shaker, 


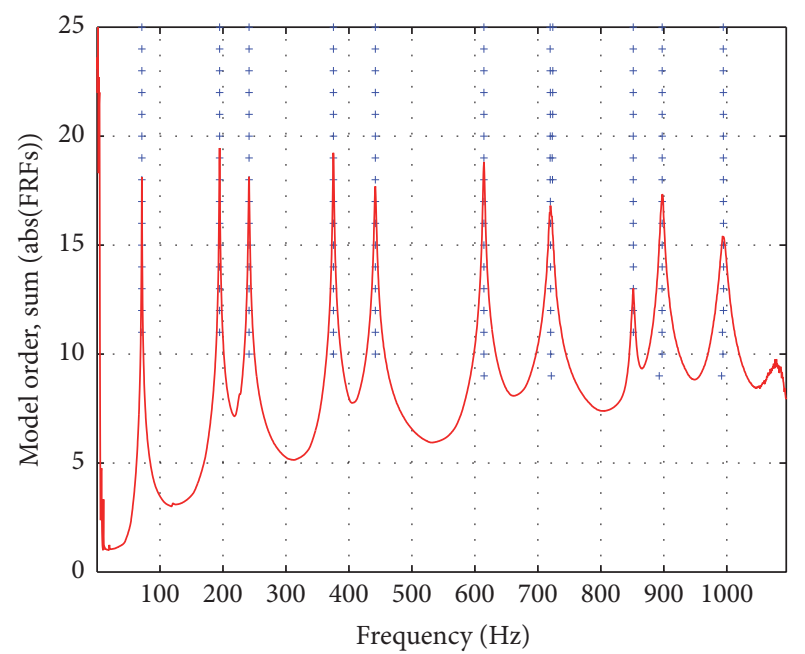

FIGURE 6: Stabilization diagram for the natural frequencies. Each “+” corresponds to an estimated frequency in the selected band, given the model order $n$ (ranging from 1 to 25 ).

driven by a white noise input signal in the frequency range 2-1000 Hz. A 24-channel signal acquisition board (OROS OR38) was used to simultaneously record the responses of 23 piezoelectric accelerometers (outputs) and the force; actuation force is delivered to the beam by a load cell positioned in the right bottom corner. Considering the leaf spring dimensions, one set of properly distributed accelerometers is sufficient to investigate the vibrational behaviour of the component.

Time domain input (force) and output (acceleration) data have been processed according to the Hv estimator [16] to produce the FRFs, which are the required inputs for the LIPEZ method. The coherence functions (not reported here for the sake of brevity) confirm the validity of the experimental setup, showing local minima much lower than one only at resonances and antiresonances. Also the input spectrum is reasonably flat, except near resonances. All data can be obtained from the authors.

The unknown model order $n$, that is, the number of modes, is increased from a minimum to a maximum value (1-25) and the results are plotted in stabilization charts: Figures 6 and 7 present the stabilization diagrams of natural frequencies and damping ratios, overlaid on the sum of the moduli of all FRFs. Separating physical from computational modes is very simple as very stable frequency lines can be observed in Figure 6. A slight scatter of damping ratios is observed in Figure 7, but the modal damping is always very low $(<0.5 \%)$.

4.2. Finite Element Model. The simple geometry of the item and the detailed characterization of the material properties-described in Section 2-allowed defining an accurate FE model by using Altair Hypermesh (Optistruct ${ }^{\circledR}$ implicit solver) as specified in $[17,18]$. The FE model precisely takes into account the stacking sequence and orientation of the plies of the actual leaf spring, whose production process was strictly controlled in all its steps from the selection of the

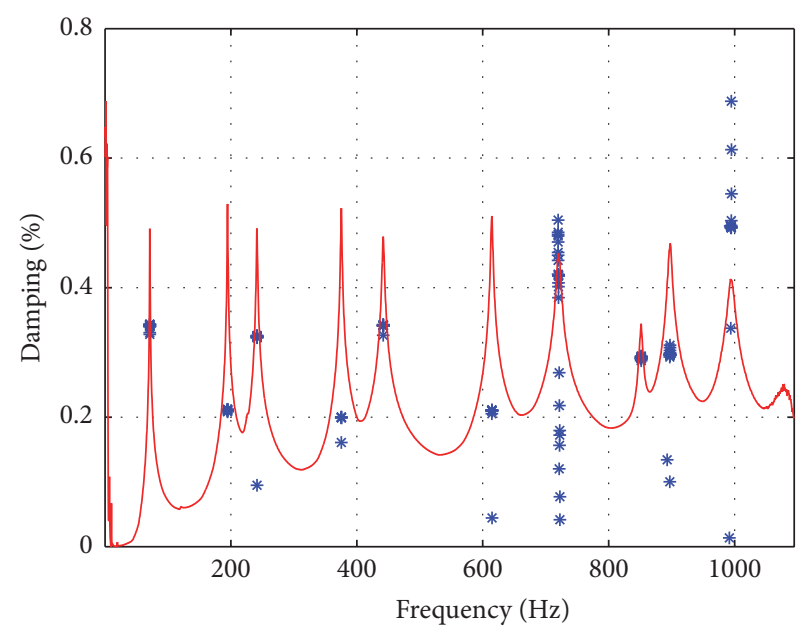

FIgURE 7: Stabilization diagram for the damping ratios. Each “*” corresponds to a pair natural frequency-damping ratio, given the model order $n$ (ranging from 1 to 25 ). For some modes, for example, mode 2 at about $200 \mathrm{~Hz}$, many points are almost completely superimposed.

materials to the final curing of the component. The 31 plies of CFRP have been modelled as a composite laminate by using the "PCOMPP" property card and the material card "MAT 8." The property is chosen because it gives the possibility of characterizing layer by layer the laminate, defining the stacking sequence, thickness, material, and orientation of each layer. The material card has been chosen because it is the only one dedicated for PCOMPP property which permits the implementation of orthotropic characteristics for shell elements as defined in $[17,18]$. Material characteristics implemented in the virtual model are defined as follows:

(i) Young modulus $E_{1}=E_{2}=44700 \mathrm{MPa}$.

(ii) $G_{12}=2500 \mathrm{MPa}$.

(iii) Poisson ratio $v_{12}=0.03$.

(iv) Density $\rho=1460 \mathrm{~kg} / \mathrm{m}^{3}$.

(v) Loss factor $\eta=0.004$.

The component is meshed with about 7000 shell elements, with mean shell size of $5 \mathrm{~mm}$, which well subdivide the entire surface of the component. The elements dimension has been properly defined by means of convergence test, not reported for the sake of brevity [19]. Shell elements have been chosen because of model complexity reduction considering that stresses distribution along the thickness can be neglected. No constraints are applied to the model to simulate a free-free condition replicating the real test.

It is important to point out that the first six vibrating modes (at almost zero frequency) describe the rigid body motion and are not be taken into account in the following analysis.

The Lanczos algorithm [20, 21] has been used to solve the undamped eigenvalue problem; the damping matrix is disregarded not only for limiting the numerical issues, but also because as proven by the Oberst beam test and 
TABLE 2: Natural frequencies, damping ratios, and MAC values for the leaf spring.

\begin{tabular}{|c|c|c|c|c|c|}
\hline Mode number & $f_{N}(\mathrm{~Hz})$ & $f_{E}(\mathrm{~Hz})$ & $\Delta f(\%)$ & $\zeta_{E}(\%)$ & Diag. (MAC) \\
\hline 1 & 67.80 & 71.3 & -4.9 & 0.34 & 0.98 \\
\hline 2 & 188.4 & 194.7 & -3.2 & 0.21 & 0.97 \\
\hline 3 & 238.3 & 241.4 & -1.3 & 0.32 & 0.97 \\
\hline 4 & 370.8 & 375.4 & -1.2 & 0.20 & 0.96 \\
\hline 5 & 452.4 & 442.0 & 2.6 & 0.34 & 0.96 \\
\hline 6 & 614.6 & 614.4 & 0.0 & 0.21 & 0.95 \\
\hline 7 & 748.8 & 720.2 & 4.0 & 0.38 & 0.93 \\
\hline 8 & 828.3 & 851.6 & -2.7 & 0.29 & 0.84 \\
\hline 9 & 919.8 & 897.4 & 2.5 & 0.30 & 0.91 \\
\hline 10 & 1053.9 & 994.5 & 6.0 & 0.49 & 0.87 \\
\hline
\end{tabular}

the experimental modal analysis, the damping capability of the CFRP is in fact very limited. The damping matrix should anyway be simply described by the proportional damping model because in this configuration damping can be attributed to the inherent properties of the material.

4.3. Comparison. To evaluate the correlation between the numerical analysis and the experimental results, the Modal Assurance Criterion (MAC) is adopted as described in $[5,6]$ :

$$
\operatorname{MAC}_{T S}=\frac{\left|\left\{\psi_{N}\right\}^{T}\left\{\psi_{E}\right\}^{*}\right|^{2}}{\left(\left\{\psi_{N}\right\}^{T}\left\{\psi_{N}\right\}^{*}\right)\left(\left\{\psi_{E}\right\}^{T}\left\{\psi_{E}\right\}^{*}\right)},
$$

where $\left\{\psi_{N}\right\}$ and $\left\{\psi_{E}\right\}$ indicate numerical and experimental mode shapes, respectively. This index indicates the similarity between the modes shapes and is equal to one when two compared modes have exactly the same shape. Figure 8 gives a pictorial representation of the MAC matrix, while Table 2 presents the numerical values on the main diagonal. It can be noticed that the minimum value is a very good $(0.84$ for the first 10 analyzed modes). The same table also lists the experimental and numerical natural frequencies, showing a quite good correlation. As expected, modal damping ratios are very limited.

This first experiment validates the five steps of the design procedure:

(1) Characterize the materials.

(2) Carefully control the production of the component, especially the orientation of the fibres.

(3) Build a FEM which precisely reproduces the actual geometry and stacking sequence.

(4) Experimentally extract the modal parameters.

(5) Compare the model with the experimental results.

All of these steps are necessary to fulfil the request of a reliable model, as clearly pointed out by the example described in the following chapter.

\section{Car Door Case}

The same design procedure has been followed on a more sophisticated test case: a CRFP car door panel.

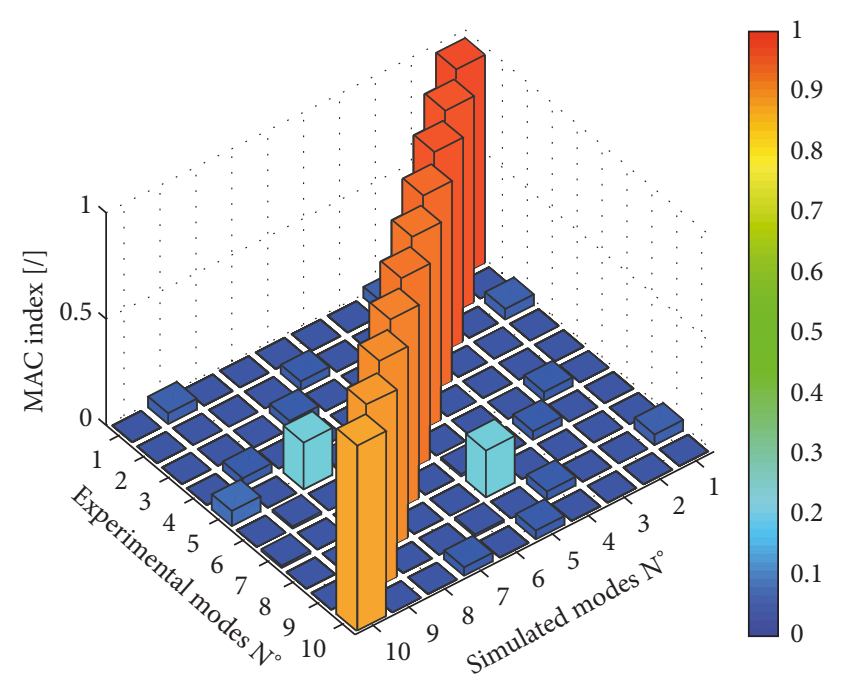

FIGURE 8: MAC coefficient for the leaf spring test.

In modern light weight design for vehicles, doors are formed by two separately produced thin shells of CFRP material, which are then tightly bonded along their outer borders. The final structure is extremely lightweight, but because of its relatively large and flat flexible surface, the influence on the acoustic characteristics of the vehicle is significant. In order to decrease the sound emission of the structure, a thin layer of damping material has been inserted along the stacking sequence, mainly for two reasons: first of all, it has to be stressed that the thin damping material is positioned on the same mould as the other carbon fibre layers and undergoes the same curing process as the standard CFRP material. A single (proper) production sequence has to be performed for both the $\mathrm{CF}$ and the damping layers so that, after curing the two separated panels, there is no need to handle again the two shells and the total cost can be reduced. The second reason is that the increment of the damping properties of the assembly is more controllable, because the constrained layer solution (CFRP-damper-CFRP) performs much better than the free layer solution (CFRP-damper).

In this section the comparison of numerical and experimental results of the external shell of a door panels are 


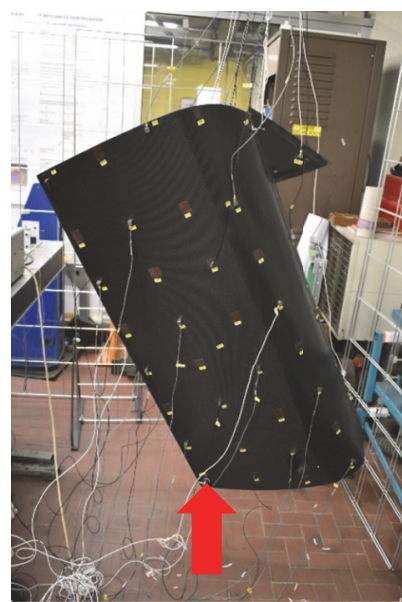

FIGURE 9: The door panel: experimental configuration with 23 accelerometers, a load cell (red arrow), and 44 measurement points.

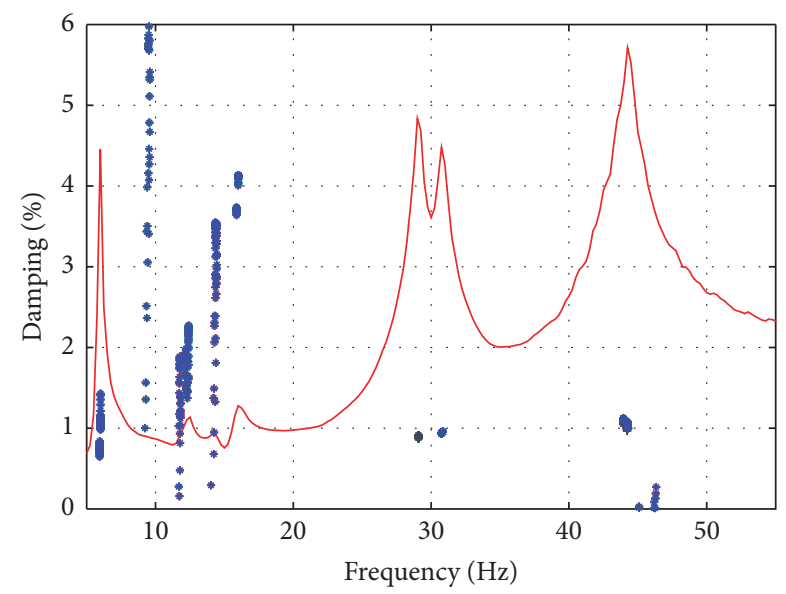

FIGURE 10: The door panel: stabilization diagram for the damping ratios: each “*” corresponds to a pair natural frequency-damping ratio.

presented with or without the integrated damping material layer.

5.1. Experimental Tests. The external shell of the door was tested in free-free conditions, with a white noise excitation force given through an electromagnetic shaker. Again, 23 accelerometers and a load cell were used to record the response but in this case two repetitions were needed to measure 44 points, aiming at a good mode shapes definition (Figure 9). The load cell was fixed on the left bottom corner and a white noise excitation was generated in the $0-400 \mathrm{~Hz}$ band. The procedure followed for data acquisition is the same as for the leaf spring and again coherence remains almost equal to one in all the analyzed frequency range, thus confirming the quality of the measured data.

Some spurious (numerical) modes are extracted by the LIPEZ method and they were simply detected by checking the stabilization diagram of damping ratios (Figure 10): numerical and nonacceptable solutions correspond to unstable or unrealistically high damping ratios, that is, the values in the $8-16 \mathrm{~Hz}$ band in Figure 10.

The modal parameters extraction has been limited to the frequency range $4-130 \mathrm{~Hz}$ which contains 11 modes.

5.2. Finite Element Model. The shell of the door panel is a handmade component of CFRP layers but its production model has been quality controlled, to ensure an actual orientation of the fibres as accurate as possible. Also the thickness of the shell has been carefully measured to limit the undesired uncertainty which could occur in proximity of small curvature radii.

The FE models are defined to reproduce the real components, with the same materials described in Section 2. A summary of the FEM main characteristics is as follows:

(i) CFRP T300 material.

(ii) Orthotropic CFRP laminate, $[0 / 90]_{3}$.

(iii) Free-free condition (no model constraints).

(iv) Element type: shell.

(v) Maximum mesh size: $4 \mathrm{~mm}$.

(vi) Eigenproblem solver: Lanczos algorithm.

5.3. Comparison. The visual comparison, confirmed by the values of the MAC matrix and the natural frequencies, shows a really poor agreement between numerical and experimental mode shapes. For example, Figure 11 gives a graphical representation of the MAC matrix, which is disappointing.

Since the material has been characterized according to the procedure described in Section 2 (which leads to an excellent model for the leaf spring) and the experimental results are very stable and reliable, the issue may be related to the aspect that does not match the requirements of the design sequence listed in Section 4.3.

The ideal disposition of the CF layers has accurately been respected at the production stage and later reproduced by the FE model, but the geometry was obtained by a sort of "reverse engineering" process. Unfortunately, a complete mathematical model of the mould is not available so that its surface has been scanned by a laser. The measured points form a basis for the ensuing CAD representation which, in turn, is the basis for the FE model. It is then reasonable to attribute the differences between numerical and experimental results to an inaccurate geometry of the FE model. A detailed revision of the numerical model has then been carried out, with particular attention to a correct definition of the curvature of the panels. The macroscopic difference is almost negligible; for example, the mass variation is limited to a mere $0.14 \%$ but the refinement is essential to tune the dynamic properties of the model and cope with the experimental results. The MAC matrix represented in Figure 12 is still not as good as expected but at least for the first four modes the coefficients (main diagonal) are above $90 \%$, confirming the impression given by the visual inspection of the mode shapes (Figure 13). Differences are still present and can be attributed to both experimental and numerical issues: the numerically redesigned geometry is not perfect yet the 


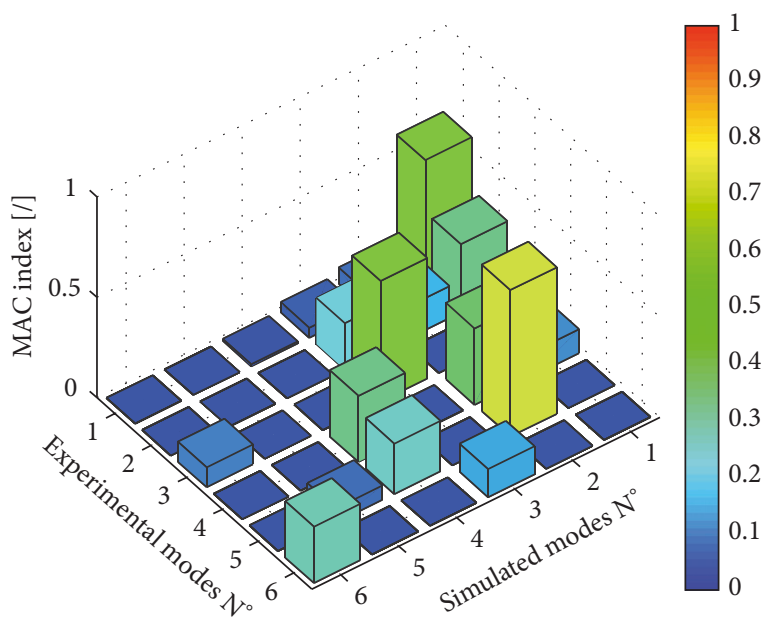

FIGURE 11: MAC coefficient for the door test, before geometry refinement.

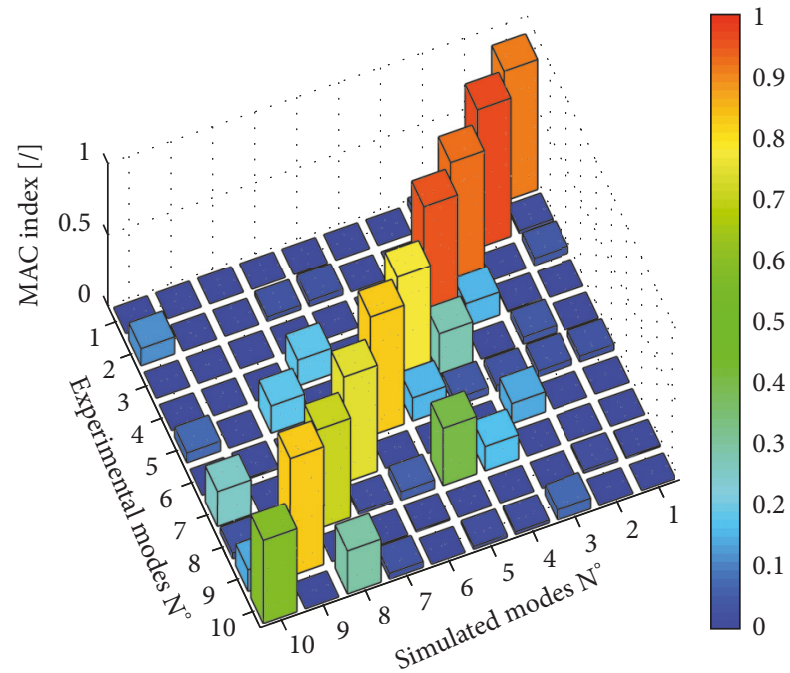

FIGURE 12: MAC coefficient for the door test, after geometry refinement.

orientation of the carbon fibres is not completely identical to the real components (the CFRP door panel is made by warping the fabric on the mould; orientation is complicated for surface with corners), the directions assigned to the measured acceleration (which are used to define the 3D mode shapes on the basis of the identified eigenvectors $\left\{\psi_{E}\right\}$ ) have inaccurately been measured, and the mass of accelerometers, load cell, and cables is not negligible with respect to the structure and also moves the system away from the ideal freefree conditions.

The conclusion is that the exact definition of the geometry and the disposition of the fibres is compulsory for a correct simulation of the dynamic behaviour of such extended and lightweight structures. The simple check on the weight of the component and its qualitative visual examination are not sufficient to accept the model, even if it is apparently very simple.
TABLE 3: Overall value of the FRFs for door panels.

\begin{tabular}{lccc}
\hline & $0-100(\mathrm{~Hz})$ & $0-200(\mathrm{~Hz})$ & $0-400(\mathrm{~Hz})$ \\
\hline Original $(\mathrm{dB})$ & 106,1 & 117,8 & 123,4 \\
Damped $(\mathrm{dB})$ & 103,0 & 110,8 & 115,1 \\
\hline
\end{tabular}

5.4. Damped versus Undamped Configuration. This section compares the FRFs of the external shell of the door with and without an embedded layer of damping material. The original shell is formed by six CF layers, with total mass equal to $2.09 \mathrm{~kg}$, which are almost unable to dissipate vibration energy as pointed out by the low values modal damping ratios (about 1\%, see Figure 10). A similar shell has been manufactured (same material and same orientation of the fibres) with a 31-3 stacking sequence: the inner core is made of a KRAIBON SUT9609/24 damping layer which undergoes the same curing cycle as the CFRP. The SUT9609/24 damping material is divided into two patches as sketched in Figure 14 and the total mass of the damped door is $2.24 \mathrm{~kg}$, a very limited increment with respect to the undamped configuration.

The two shells underwent the same experimental tests and 44 FRFs were measured (see Figure 9) by using a random input in the band $0-400 \mathrm{~Hz}$. The effect of the damping material is obvious when listening to the sound emitted by the two panels (undamped and damped). A quantification of the effectiveness of the constrained layer is given in Figure 15 where the sum of the moduli of all 44 FRFs (inertance) is plotted: the red dotted line (no damping) is well above the solid blue curve along the entire testing frequency range, especially for frequency higher than $50 \mathrm{~Hz}$ (which is important for limiting the interior noise harshness).

Table 3 gives the overall value of the FRFs, that is, the sum of the modals extended over a certain frequency range, for the two configurations. Even in the $0-100 \mathrm{~Hz}$ region the difference is above $3 \mathrm{dBs}$, that is, half-power, and furtherly increases with the frequency band.

\section{Conclusions}

Structural material properties have been determined by the Oberst beam technique; a database which permits reliable reproduction of experimental tests by a FE model has been defined. Both pure CFRP structures and sandwich structures formed by CRFP and a damping material have been tested in the temperature range $-20 /+60^{\circ} \mathrm{C}$ to evaluate the variability of Young's modulus and the loss factor. A couple of CFRP automotive components, a leaf spring and the external panel of a door, have been manufactured and tested. The comparison of the experimental modal analysis with the results of the FE models revealed the two extreme important aspects. Not only has the material to be correctly defined but also a precise geometry of the FE model should be created to achieve a good correlation: even small geometrical variations, especially in curved and large surfaces, can lead to significant differences for dynamic responses. The influence of damping material has experimentally been verified and quantified on the door panel. A thin layer of KRAIBON SUT9609/24 damping material has been embedded in the stacking sequence of 

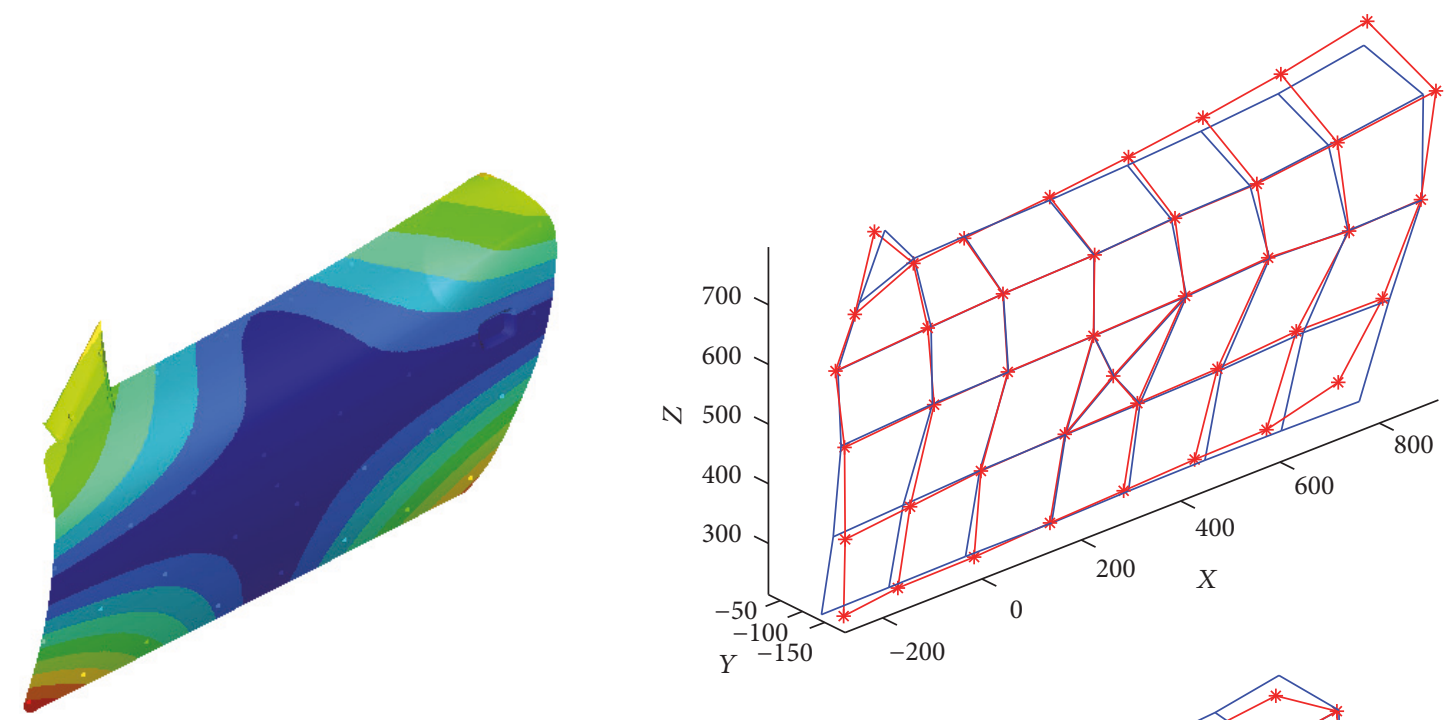

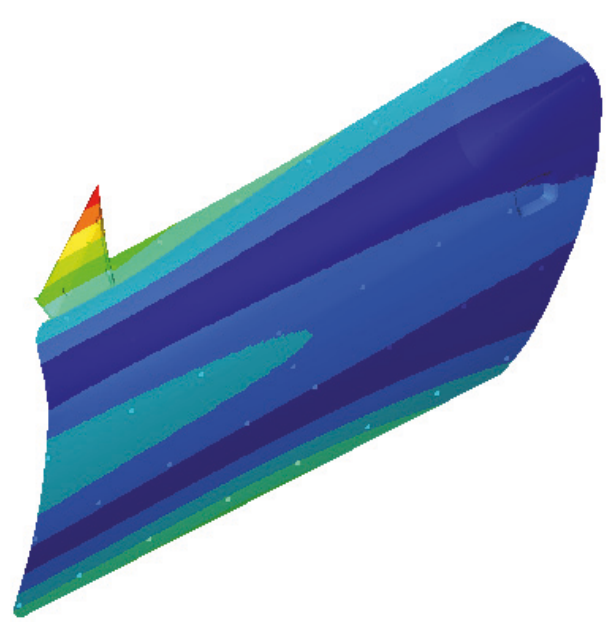

(a)

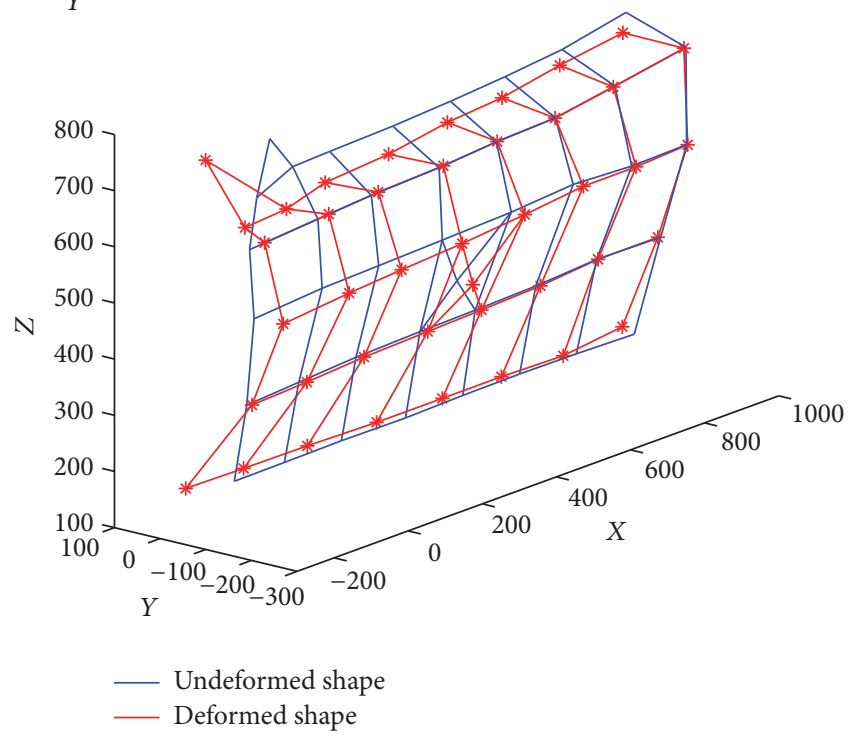

(b)

FIGURE 13: The first two mode shapes after the geometry refinement: (a) FEM; (b) experimental.

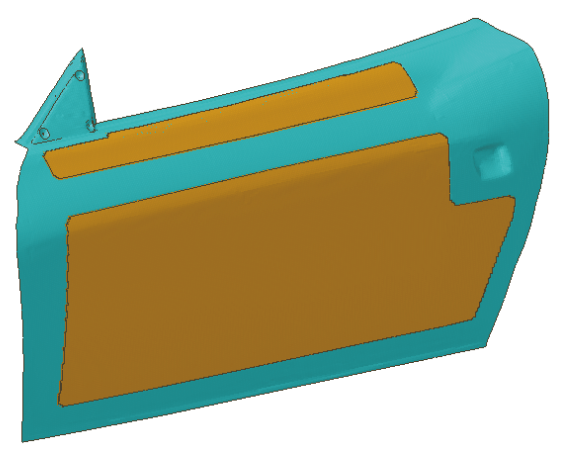

Figure 14: Comparison of the damped and undamped panels: position of the damping patches.

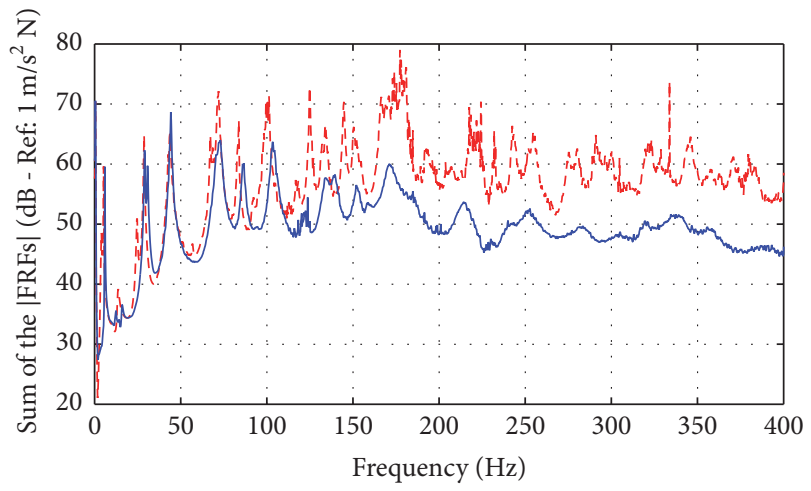

Figure 15: Comparison of the damped and undamped panels: sum of the FRFs: red dashed line: undamped panel-blue solid line: damped panel. 
the panel, with very limited impact on both the production process and the final cost of the component. The weigh variation of the panel is within $+7 \%$ but its dynamic response dramatically improved with potentially significant effects on the noise harshness of the car interior environment.

\section{Conflicts of Interest}

The authors declare that there are no conflicts of interest regarding the publication of this paper.

\section{Acknowledgments}

The authors wish to acknowledge Altair-Italy Engineering S.R.L. for providing the FE software and Gummiwerk KRAIBURG GmbH for the damping material "KRAIBON SUT9609/24" and the active support during all the activity and G.Angeloni ${ }^{\circledR}$ S.R.L. for the structural CFRP material.

\section{References}

[1] M. Carello and A. G. Airale, "Composite Suspension Arm Optimization for the City Vehicle XAM 2.0," in Design and Computation of Modern Engineering Materials, A. Öchsner and H. Altenbach, Eds., vol. 54, pp. 257-272, Cham: Springer International Publishing, 2014.

[2] M. Carello, A. Airale, A. Ferraris, and A. Messana, "XAM 2.0: from Student Competition to Professional Challenge," Computer-Aided Design and Applications, vol. 11, pp. S61-S67, 2014.

[3] M. Carello, A. G. Airale, and A. Ferraris, "City vehicle XAM 2.0: design and optimization of the composite suspension system," SAE Technical Papers, vol. 1, pp. 1-10, 2014.

[4] G. Brusaglino, G. Buja, M. Carello, A. P. Carlucci, C. H. Onder, and M. Razzetti, "New technologies demonstrated at Formula Electric and Hybrid Italy 2008," World Electric Vehicle Journal, vol. 3, no. 1, pp. 1-12, 2009.

[5] H. Wan, Y. Li, and L. Zheng, "Vibration and damping analysis of a multilayered composite plate with a viscoelastic midlayer," Shock and Vibration, vol. 2016, Article ID 6354915, 10 pages, 2016.

[6] Standard., Standard Test Method for Measuring VibrationDamping Properties of Materials, ASTM International, 2010.

[7] G. Erdoğan, F. Bayraktar, and K. Y. Sanliturk, "Measurement of Dynamic Properties of Materials," in Proceedings of the 32nd International Congress and Exposition on Noise Control Engineering, Jeju International Convention Center, Seogwipo, Korea, August 2003.

[8] J1637: Laboratory Measurement of the Composite Vibration Damping Properties of Materials on a Supporting Steel Bar SAE International. http://standards.sae.org/j1637_201306/.

[9] N. M. M. Maia and J. M. Montalvão e, Eds., Theoretical And Experimental Modal Analysis, Research Studies Press, New York, NY, USA, 1997.

[10] D. J. Ewins, Modal Testing: Theory, Practice, and Application, Research Studies Press, Philadelphia, PA, USA, 2nd edition, 2000.

[11] E. Gandino, L. Garibaldi, and S. Marchesiello, "Covariancedriven subspace identification: a complete input-output approach," Journal of Sound and Vibration, vol. 332, no. 26, pp. 7000-7017, 2013.

[12] B. Peeters, H. Van Der Auweraer, P. Guillaume, and J. Leuridan, "The PolyMAX frequency-domain method: a new standard for modal parameter estimation?" Shock and Vibration, vol. 11, no. 3-4, pp. 395-409, 2004.

[13] J. Wang, C. Wang, T. Zhang, and B. Zhong, "Comparison of different independent component analysis algorithms for output-only modal analysis," Shock and Vibration, vol. 2016, Article ID 6309084, pp. 1-25, 2016.

[14] A. Fasana, "Modal parameters estimation in the Z-domain," Mechanical Systems and Signal Processing, vol. 23, no. 1, pp. 217225, 2009.

[15] A. Fasana, M. Carello, A. Ferraris, A. Airale, and D. B. Polato, "NVH Analysis of Automotive Components: A Carbon Fiber Suspension System Case," in Advances in Italian Mechanism Science, G. Boschetti and A. Gasparetto, Eds., vol. 47, pp. 345354, Springer International Publishing, 2017.

[16] K. Shin and J. K. Hammond, Fundamentals of signal processing for sound and vibration engineers, John Wiley and Sons, Hoboken, NJ, USA, 2008.

[17] Altair Engineering, Altair Engineering: Practical Aspect of Finite Element Simulation, 3rd edition, 2015.

[18] Altair Engineering, Altair engineering: Pre and post - processing for Finite Element Analysis, guidebook, 2015.

[19] J. Jin and D. J. Riley, "Effect of mesh size on finite element analysis of plate structure," International Journal of Engineering Science and Innovative Technology, pp. 181-185, 2015.

[20] J. Rydh, Very Large Symmetric Eigenvalue Problems Solved by Lanczos and Multiple Relatively Robust Representations for Tridiagonals, School of Engineering Physics, Royal Institute of Technology, 2007.

[21] Combining automated multilevel substructuring and subspace iteration for huge gyroscopic eigenproblems, ResearchGate, https://www.researchgate.net/publication/259193426_Combining_automated_multilevel_substructuring_and_subspace_iteration_for_huge_gyroscopic_eigenproblems. 


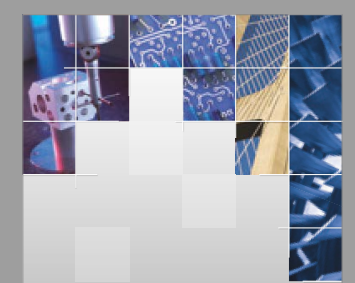

\section{Enfincering}
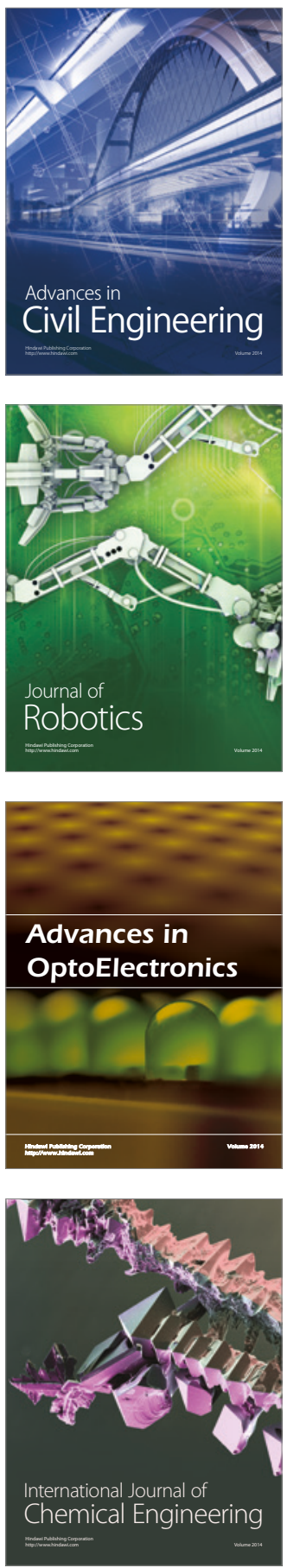

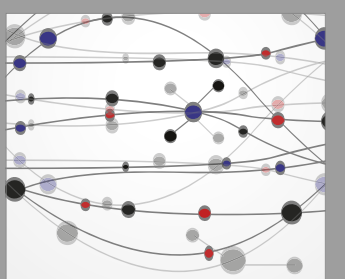

The Scientific World Journal

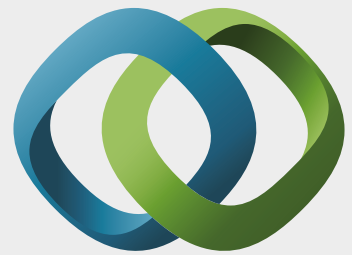

\section{Hindawi}

Submit your manuscripts at

https://www.hindawi.com
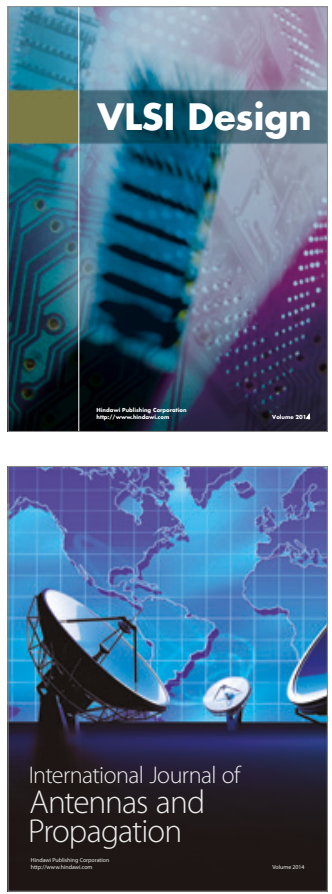

\section{Rotating}

Machinery
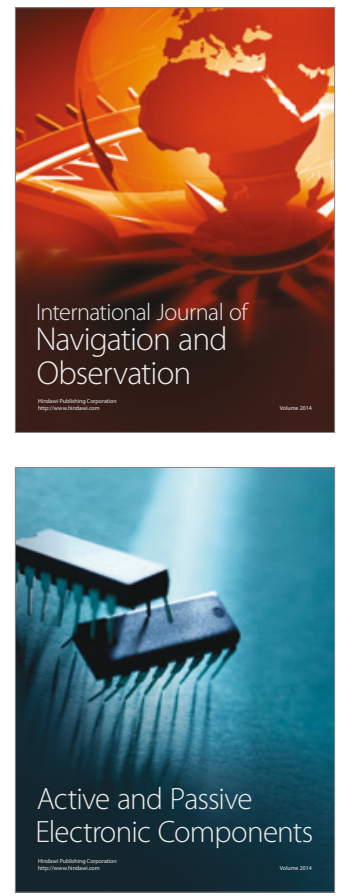
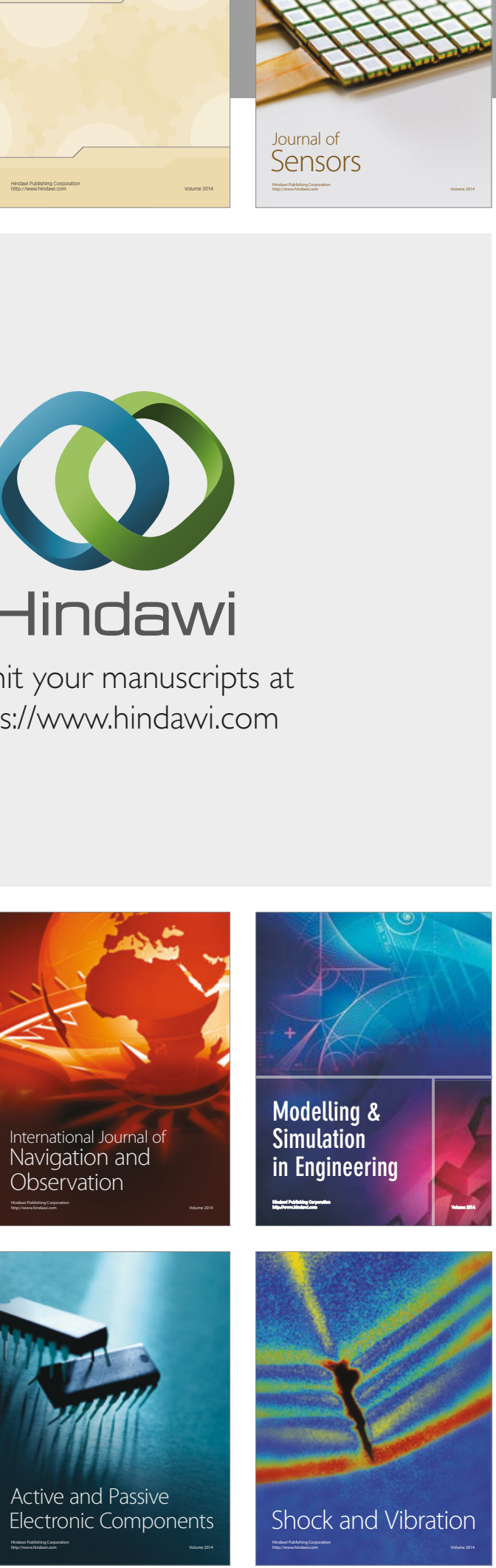
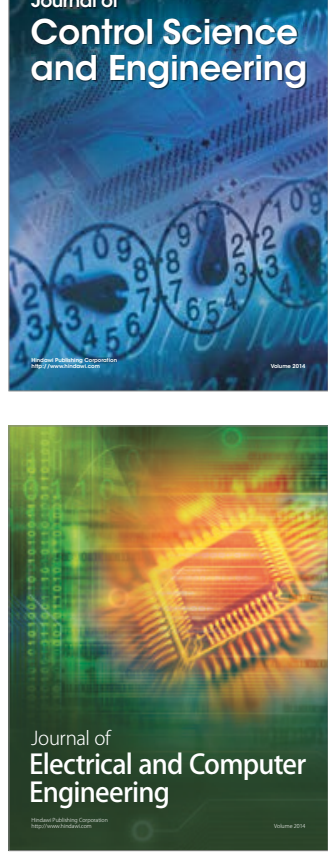

Distributed

Journal of

Control Science

and Engineering
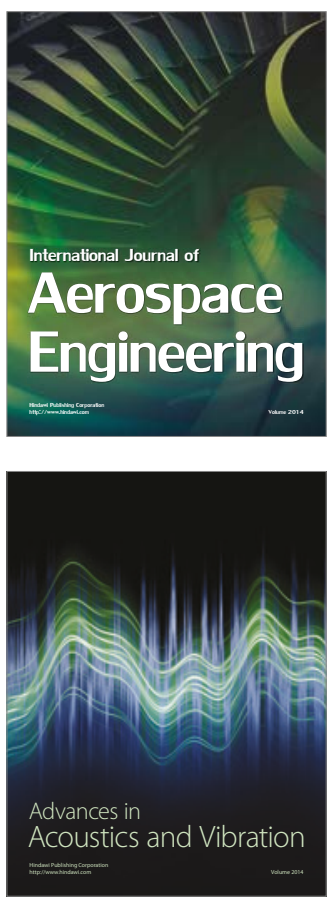

Sensor Networks 